\title{
Functionalized $\mathrm{N}, \mathrm{N}$-Diphenylamines as Potent and Selective EPAC2 Inhibitors
}

\author{
Christopher T. Wild, ${ }^{\dagger, \S}$ Yingmin Zhu, ${ }^{\ddagger}$, Ye Na, $^{\dagger}$ Fang Mei, ${ }^{\ddagger}$ Marcus A. Ynalvez, ${ }^{\dagger}$ Haiying Chen, ${ }^{\dagger}$ \\ Xiaodong Cheng, ${ }^{*}+$ and Jia Zhou* ${ }^{*} \dagger$ \\ ${ }^{\dagger}$ Chemical Biology Program, Department of Pharmacology and Toxicology, University of Texas Medical Branch, Galveston, Texas \\ 77555, United States \\ ${ }^{\ddagger}$ Department of Integrative Biology and Pharmacology and Texas Therapeutics Institute, University of Texas Health Science Center, \\ Houston, Texas 77030, United States
}

\section{Supporting Information}

\begin{abstract}
N, N$-Diphenylamines were discovered as potent and selective EPAC2 inhibitors. A study was conducted to determine the structure-activity relationships in a series of inhibitors of which several compounds displayed submicromolar potencies. Selectivity over the related EPAC1 protein was also demonstrated. Computational modeling reveals an allosteric site that is distinct from the cAMP binding domain shared by both EPAC isoforms, providing a theory with regards to subtype selectivity.
\end{abstract}

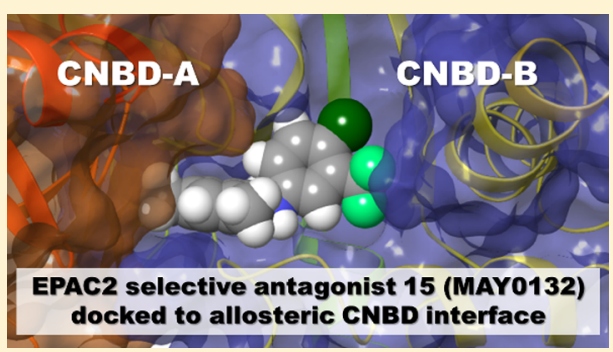

KEYWORDS: Exchange proteins directly activated by cAMP (EPAC) inhibitors, diphenylamines, Buchwald-Hartwig amination, structure-activity relationship

$\mathrm{T}$

he intracellular effects of cyclic adenosine monophosphate (cAMP) are mediated by intracellular effector proteins with evolutionary conserved cAMP binding domains (CNBD). ${ }^{1}$ Prior to the discovery of exchange proteins directly activated by cAMP (EPAC) in 1998, cAMP-mediated signaling events were believed to be transduced largely by protein kinase A (PKA). ${ }^{2,3}$ The discovery of EPAC has changed the landscape of cAMPsignaling research. Evidence continues to emerge underscoring the importance of EPAC and its complex relationship to PKA. $^{4-6}$ Upon binding cAMP, EPAC proteins activate the Ras superfamily small GTPases Rap1 and Rap2. ${ }^{2,3}$ To date, many studies have shown the physiological and pathophysiological significance of EPAC proteins ${ }^{7}$ and their involvement in cancer, ${ }^{8,9}$ bacterial and viral infections, ${ }^{10,11}$ energy homeostasis and obesity, ${ }^{12,13}$ as well as cardiac functions. ${ }^{14,15}$ Given that mammalian EPAC proteins exist as two structurally homologous but functionally nonredundant isoforms, EPAC1 and EPAC2, ${ }^{16,17}$ the development of isoform selective EPAC inhibitors as molecular probes and potential therapeutics is urgently needed. Herein we report the design, synthesis, pharmacological characterization, and molecular docking results of functionalized $N, N$-diphenylamines as potent and selective EPAC2 inhibitors.

Our previous high-throughput screening (HTS) campaign led to the discovery of several validated hits with specific EPAC inhibitory activity and was subsequently followed by extensive medicinal chemistry optimizations. ${ }^{9,18-21}$ Among the identified "hits", the diarylsulfone and arylsulfonamide scaffolds related to ESI-05 (1, Figure 1) were explored to elucidate structureactivity relationships (SARs) as described in our previous

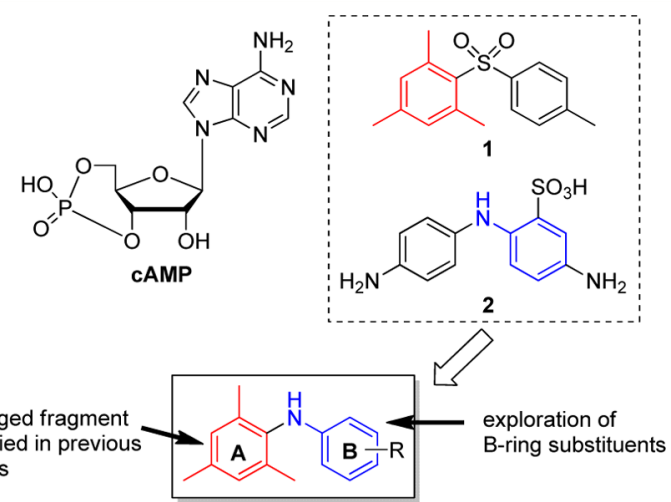

Figure 1. Structures of cAMP, HTS hits ESI-05 (1) and ESI-10 (2), and designed $\mathrm{N}, \mathrm{N}$-diphenylamines as a new class of EPAC2 inhibitors.

work. ${ }^{22}$ Herein, we present the synthesis and biological activity of $N, N$-diphenylamines that are structurally related to both HTS hits ESI-05 (1) and ESI-10 (2) (Figure 1). The general approach as depicted in Figure 1 was to retain the 2,4,6trimethylphenyl (mesityl) moiety or A-ring of 1, a favorable hydrophobic fragment of EPAC antagonists identified in previous studies. The incorporation of various electron withdrawing and donating groups with differing substitution patterns in the B-ring of the diphenyl amine was used to

Received: December 10, 2015

Accepted: March 28, 2016

Published: March 28, 2016 
explore the B-ring electronic requirements to optimize EPAC2 inhibitory activity and selectivity. To this end, a one-step synthetic strategy based on the Buchwald-Hartwig amination protocol $^{7}$ was utilized as a facile entry to potential EPAC2 inhibitors (Scheme 1, 3-32). B-ring fragments were chosen

Scheme 1. Synthesis of $N, N$-Diphenylamine Scaffold ${ }^{a}$
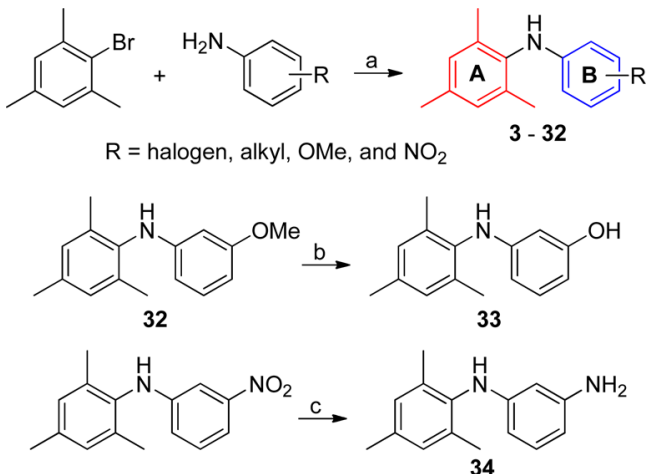

${ }^{a}$ Reagents and conditions: (a) $\mathrm{Pd}_{2}(\mathrm{dba})_{3}, \mathrm{NaO}^{t} \mathrm{Bu},( \pm)$-BINAP, toluene, $120{ }^{\circ} \mathrm{C}, 11-97 \%$; (b) $48 \% \mathrm{HBr}$ (aq), $110{ }^{\circ} \mathrm{C}, 50 \%$; (c) $\mathrm{H}_{2}$, $\mathrm{Pd} / \mathrm{C}, \mathrm{MeOH}, 74 \%$.

based on our previous findings, ${ }^{17,19,22,23}$ commercial availability, and coupling capacity in the described protocol. The demethylation of methoxy and reduction of nitro groups were achieved utilizing standard protocols to afford 33 and 34, respectively. The synthetic protocols and detailed characterization data are reported in the Supporting Information (SI).

A previously described EPAC2-based assay utilizing a fluorescent cyclic nucleotide analogue 8-NBD-cAMP was used to quantify the test ligands' capacity to prevent 8-NBDcAMP binding to EPAC2. ${ }^{17,18}$ The binding of 8-NBD-cAMP to purified EPAC2 leads to a dose-dependent increase in fluorescent signal, which can be reversed by cAMP or EPAC2 antagonists. Therefore, fluorescence intensity can be used to quantify the potency of the synthesized compounds as the $\mathrm{IC}_{50}$ values (Table 1). Our previous report disclosed HJC0338 (Table 1), a diphenylamine that was able to prevent 8-NBDcAMP binding to EPAC2. This provided the rationale to further investigate a larger number of analogues. ${ }^{22}$ Therefore, dichloro analogues were synthesized and assessed to have promising activity similar to previously reported compounds, e.g., 2,3-dichloro derivative $\left(4, \mathrm{IC}_{50}=0.7 \mu \mathrm{M}\right){ }^{22}$ Incorporation of a methyl into the B-ring proved beneficial as shown by 12 (3chloro-2-methyl, $\left.\mathrm{IC}_{50}=0.5 \mu \mathrm{M}\right)$. Incorporation of fluorine, bromine, and trichloro/trifluoro provided little to no increase in activity $(6-10,16-21)$. Inclusion of trifluoromethyl resulted in active compounds. As an example, 15 demonstrated EPAC2 inhibition $\left(\mathrm{IC}_{50}=0.4 \mu \mathrm{M}\right)$. While incorporation of a single methyl, trifluoromethyl, ethyl, dimethyl, and other hydrophobic moieties showed virtually no gains in inhibitory activity (2226, 28-30). The 3,5-bis(trifluoromethyl) derivative 27 and 2,4,6-trimethyl derivative 31 both displayed promising inhibitory activities ( $\mathrm{IC}_{50}=0.6$ and $0.4 \mu \mathrm{M}$, respectively). Incorporation of the electron donating 3-methoxy, 3-hydroxy, and 3-amino groups (33-34) provided no gain in activity. Based on the obtained SAR, hydrophobicity of both phenyl rings remains essential for inhibitory activity, consistent with our previously reported EPAC2 antagonists. ${ }^{17,19}$
Table 1. $\mathrm{IC}_{50}$ Values of Functionalized $N, N$-Diphenylamine Scaffolds in 8-NBD-cAMP EPAC2 Binding Assay

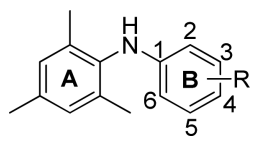

\begin{tabular}{|c|c|c|}
\hline compd & $\mathrm{R}$ & $\mathrm{IC}_{50}(\mu \mathrm{M})^{a}$ \\
\hline $\mathrm{cAMP}^{b}$ & see Figure 1 & 40 \\
\hline $1^{b}$ & see Figure 1 & 0.5 \\
\hline $2^{b}$ & see Figure 1 & 18 \\
\hline $\mathrm{HJC} 0338^{b}$ & 2,5-dichloro & 0.4 \\
\hline 3 & 3,4-dichloro & $1.1 \pm 0.3$ \\
\hline 4 & 2,3-dichloro & $0.7 \pm 0.2$ \\
\hline 5 & 2,4-dichloro-6-methyl & $0.7 \pm 0.3$ \\
\hline 6 & 3,5-difluoro & $5.7 \pm 1.3$ \\
\hline 7 & 3,4-difluoro & $8.7 \pm 1.7$ \\
\hline 8 & 3-chloro-5-fluoro & $2.9 \pm 0.5$ \\
\hline 9 & 4-chloro-3-fluoro & $3.3 \pm 0.6$ \\
\hline 10 & 3-chloro-4-fluoro & $2.9 \pm 0.5$ \\
\hline 11 & 3-chloro-4-methyl & $1.8 \pm 0.3$ \\
\hline 12 & 3-chloro-2-methyl & $0.5 \pm 0.2$ \\
\hline 13 & 5-chloro-2-methyl & $1.4 \pm 0.4$ \\
\hline 14 & 3-chloro-5-trifluoromethyl & $0.5 \pm 0.1$ \\
\hline 15 & 4-chloro-3-trifluoromethyl & $0.4 \pm 0.1$ \\
\hline 16 & 3-bromo & $1.5 \pm 0.4$ \\
\hline 17 & 4-bromo & $1.6 \pm 0.4$ \\
\hline 18 & 3-fluoro & $5.8 \pm 1.1$ \\
\hline 19 & 3,4,5-trichloro & $1.1 \pm 0.3$ \\
\hline 20 & 3,4,5-trifluoro & $3.0 \pm 0.5$ \\
\hline 21 & 2,4,6-trifluoro & $2.7 \pm 0.5$ \\
\hline 22 & 2,3-dimethyl & $1.0 \pm 0.2$ \\
\hline 23 & 2,4-dimethyl & $2.2 \pm 0.3$ \\
\hline 24 & 2,5-dimethyl & $1.8 \pm 0.3$ \\
\hline 25 & 3,5-dimethyl & $1.7 \pm 0.2$ \\
\hline 26 & $3,4-\left(\mathrm{CH}_{2} \mathrm{CH}_{2} \mathrm{CH}_{2}\right)-$ & $1.8 \pm 0.3$ \\
\hline 27 & 3,5-bis(trifluoromethyl) & $0.6 \pm 0.1$ \\
\hline 28 & 3-ethyl & $1.8 \pm 0.4$ \\
\hline 29 & 3-trifluoromethyl & $2.0 \pm 0.4$ \\
\hline 30 & 3-(mesitylethynyl)phenyl & $>300$ \\
\hline 31 & 2,4,6-trimethyl & $0.4 \pm 0.1$ \\
\hline 32 & 3-methoxy & $10.7 \pm 3.9$ \\
\hline 33 & 3-hydroxy & $46.0 \pm 7.9$ \\
\hline 34 & 3-amino & $34.0 \pm 8.7$ \\
\hline . & 22 & \\
\hline
\end{tabular}

Several promising compounds, 12, 15, 27, and 31 (Figure 2), were screened for selectivity between EPAC2 and EPAC1. A previously described ${ }^{17}$ in vitro guanine nucleotide exchange factor (GEF) functional assay was used to assess selectivity given that the 8-NBD-cAMP binding assay is only useful for EPAC2, not EPAC1 under HTS format (Table 2, Figure 3). Compounds 12, 15, 27, and 31 do not display measurable EPAC1 activity under the doses tested in the reported assay (no effect at up to $100 \mu \mathrm{M}$ ) yet display low micromolar inhibitory activity at EPAC2. However, ESI-09, a nonselective EPAC $1 / 2$ antagonist, displayed EPAC1 and EPAC2 inhibitory activity under the same conditions (Table 2, Figure 3 ). ${ }^{18}$ This suggests that the aforementioned compounds represent potent and highly selective EPAC2 inhibitors. Interestingly, while each compound inhibited EPAC2 GEF activity to the same extent ( $>80 \%$ activity ablation, Figure 3 ), the same compounds vary in their capacity to prevent 8-NBD-cAMP binding to EPAC2 


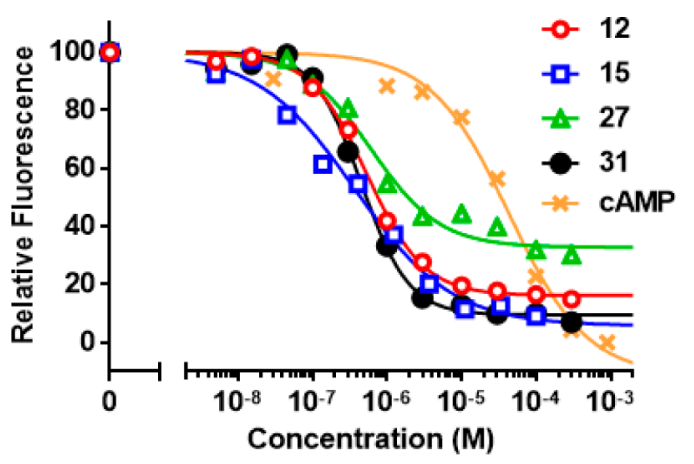

Figure 2. Relative potency of EPAC2 inhibitors 12, 15, 27, 31, and cAMP; dose-dependent inhibition of 8-NBD-cAMP binding to EPAC2; open circles, 12; open squares, 15; open triangles, 27; closed circles, $31 ; \times$, cAMP $(n \geq 3)$.

Table 2. $\mathrm{IC}_{50}$ Values of Select $N, N$-Diphenylamines in cAMP-Mediated Guanine Nucleotide Exchange Factor (GEF) Activity Assay of EPAC2 or EPAC1

\begin{tabular}{lcl} 
& \multicolumn{2}{c}{$\mathrm{IC}_{50}(\mu \mathrm{M})$} \\
\cline { 2 - 3 } compd & $\mathrm{EPAC} 2$ & $\mathrm{EPAC1}$ \\
12 & $3.6 \pm 1.0$ & $\mathrm{NE}^{a}$ \\
15 & $1.0 \pm 0.1$ & $\mathrm{NE}$ \\
27 & $1.3 \pm 0.2$ & $\mathrm{NE}$ \\
31 & $1.7 \pm 0.3$ & $\mathrm{NE}$ \\
ESI-09 & $4.4 \pm 0.5$ & $10.8 \pm 0.6$
\end{tabular}

${ }^{a} \mathrm{NE}$ (no effect at up to $100 \mu \mathrm{M}$ ). ${ }^{b}$ See ref 18 .

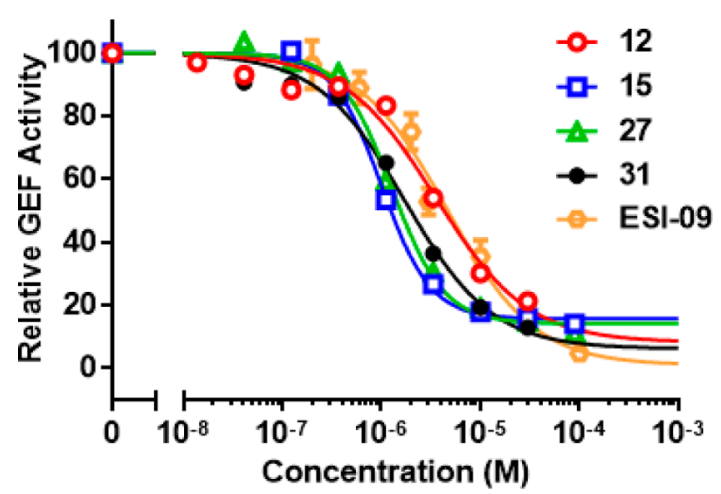

Figure 3. Relative cAMP-mediated EPAC2 GEF activity in the presence of 12, 15, 27, and 31, and ESI-09; open red circles, 12; open squares, 15; open triangles, 27 ; closed circles, 31 ; open orange hexagons, ESI-09 $(n=3)$.

(Figure 2). Compound 27 led to a decrease in 8-NBD-cAMP binding of approximately $60 \%$, while the other compounds prevent roughly $80-90 \%$ of $8-\mathrm{NBD}$-cAMP binding. However, all four compounds display nearly the same inhibitory activity in the functional assay. This suggests that the tested EPAC2 inhibitors may not only prevent 8-NBD-cAMP binding but also promote a conformation that decreases EPAC2 GEF activity.

To gain insight with regards to EPAC2 selectivity, molecular docking studies were performed using the Schrödinger Drug Discovery Suite to investigate predicted binding modes of the $\mathrm{N}, \mathrm{N}$-diphenylamine scaffold and apo-EPAC2 (PDB\# 2BYV; see Experimental Methods in the SI). ${ }^{24}$ Both EPAC1 and EPAC2 contain a conserved cAMP binding domain that binds cAMP with high affinity (CNBD-B, Figure 4). Binding of cAMP to the CNBD-B results in a conformational change of EPAC that

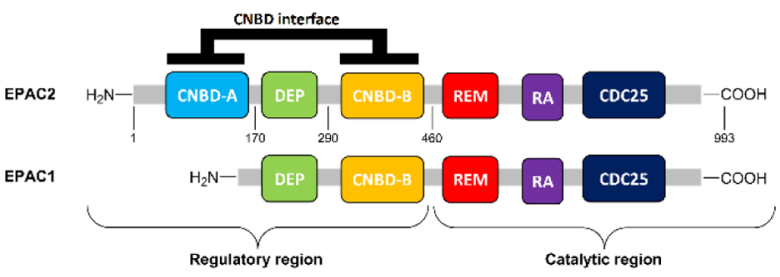

Figure 4. Domains of EPAC proteins. All EPAC family members possess an N-terminal autoinhibitory regulatory region and a $\mathrm{C}$ terminal catalytic region. The regulatory region contains a Dishevelled, Egl-10, and Pleckstrin (DEP) domain and up to two cyclic adenosine monophosphate (cAMP) nucleotide-binding domains (CNBD). While EPAC1 contains only one $\mathrm{CNBD}$ and is truncated at the $\mathrm{N}$-terminus, EPAC2 contains two cAMP binding domains (CNBD-A and CNBD$B)$, which form an interface in the tertiary structure of the autoinhibited apo-EPAC2 (PDB\# 2BYV).

exposes the catalytic region responsible for Rap activation. However, EPAC2 has an additional cAMP binding domain (CNBD-A) that binds cAMP with a low affinity and is not required for EPAC2 regulation by cAMP. ${ }^{4}$ Interestingly, in the reported autoinhibitory apo-EPAC2 structure, CNBD-A and CNBD-B are oriented toward each other forming an interface that blocks both cAMP cavities (Figure 4). ${ }^{24,25}$ EPAC1 lacks this interface, given that it only contains the CNBD-B. Therefore, it is conceivable that a small molecule can prevent the binding of cAMP (or the 8-NBD-cAMP probe used in the described assay) to EPAC2 by bridging CNBD-A and CNBD-B domains. This would promote stabilization of the autoinhibitory, versus the active state, a transition that is known to be highly dynamic for EPAC. ${ }^{25-30}$ This interaction is not possible for EPAC1 as it lacks the interface. This idea is supported by the fact that our original HTS hit $\mathbf{1}$ loses EPAC2 inhibitory activity upon deletion of CNBD-A. ${ }^{31}$ Additionally, $15(1 \mu \mathrm{M})$ acted as a noncompetitive inhibitor of cAMPmediated EPAC2 GEF activity by producing a rightward-shift in cAMP dose-response and reduction in maximum activation (see SI, Figure S1), suggesting that these, and similar scaffolds, may access an allosteric site such as the interface of CNBD-A and CNBD-B. Noncompetitive/allosteric inhibition of EPAC1 has been proposed in previous reports of ligands that act at the hinge region between CNBD-B and REM (Figure 4). However, the hinge region is highly conserved in both EPAC 1 and 2.32,33 Since compounds 12, 15, 27, and 31 selectively inhibit EPAC2 but not EPAC1, it is reasonable to suggest that the aforementioned compounds interact at the interface of both cAMP binding domains in the EPAC2 protein. Therefore, molecular docking to the apo-EPAC2 at the CNBD-A and CNBD-B interface was conducted with ligands 12, 15, 27, and 31. Lowest energy poses for each are overlaid in Figure 5 showing a well-defined binding pocket between the cAMP binding domains. A zoomed view of $\mathbf{1 5}$ and apo-EPAC2 (Figure 6) predicts a key cation $-\pi$ interaction between the electron rich A ring and LYS 42 of CNBD-A and an H-bond interaction between the $\mathrm{N}, \mathrm{N}$-diphenylamine $\mathrm{N}-\mathrm{H}$ and the backbone amide carbonyl of HIS 335 of CNBD-B. Therefore, the privileged mesityl fragment and $\mathrm{N}-\mathrm{H}$ bridge the gap between $\mathrm{CNBD}-\mathrm{A}$ and $\mathrm{CNBD}-\mathrm{B}$ in EPAC2, presumably stabilizing the autoinhibited state, a binding mode that is not possible in EPAC1 provided the absence of CNBD-A. In fact, all but one of the compounds can be docked to this interface with similar results indicating that a basic structural requirement for this predicted binding pose is the diphenylamine 


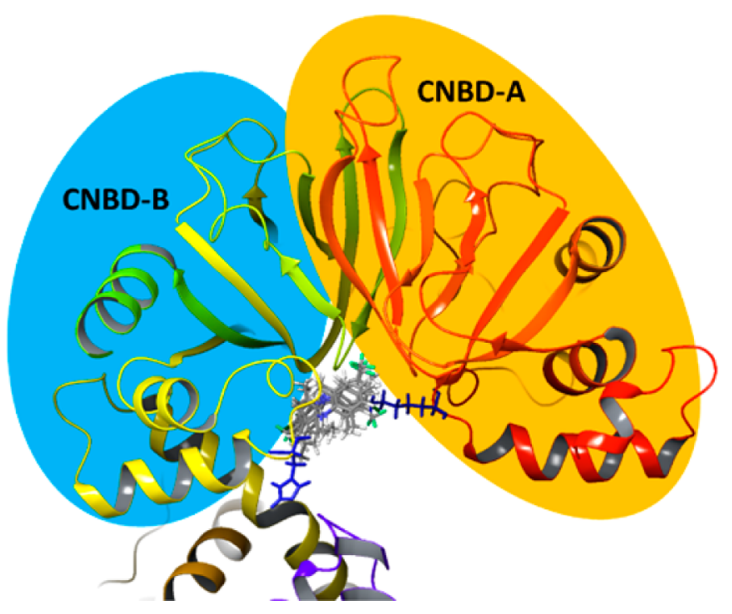

Figure 5. Lowest energy docking pose overlay of 12, 15, 27, and 31 (gray stick) with autoinhibited apo-EPAC2 (ribbon, PDB code: 2BYV); key residues LYS 42 and HIS 335 (blue stick); CNBD-A highlighted with orange ellipse; CNBD-B highlighted with light blue ellipse.

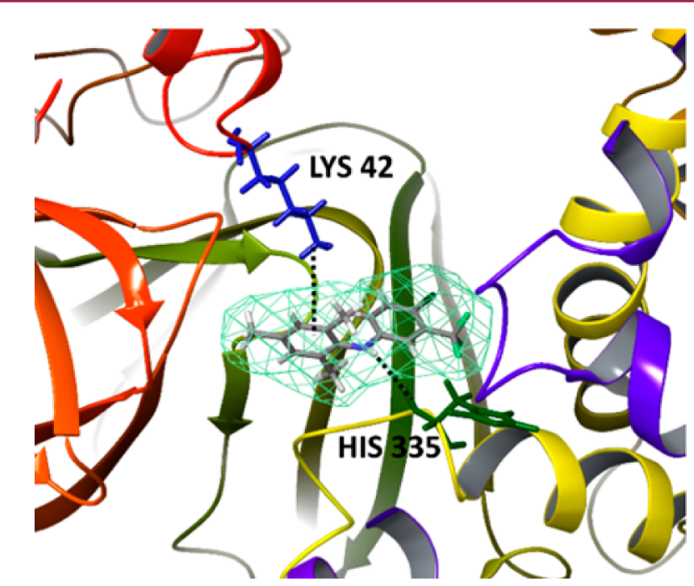

Figure 6. Zoomed-view of lowest energy docking pose of $\mathbf{1 5}$ (gray stick/wire-mesh) with autoinhibited apo-EPAC2 (ribbon, PDB \# 2BYV); key residues LYS 42 (blue stick) from CNBD-A and HIS 335 amide (green stick) from CNBD-B.

scaffold. An exception, compound 30, is not able to access the proposed allosteric site due to its size, consistent with the fact that compound 30 was ineffective in preventing 8 -NBD-cAMP binding (Table 1). Moreover, ligands with strong electron donating groups $\left(-\mathrm{OMe},-\mathrm{OH},-\mathrm{NH}_{2} ; 32-34\right)$ consistently display $\mathrm{IC}_{50}$ values weaker than the other compounds tested. Given that the docking indicates that the $N, N$-diphenylamine $\mathrm{N}-\mathrm{H}$ interacts with the amide carbonyl of HIS 335 of CNBD$\mathrm{B}$, it is conceivable that $-\mathrm{OMe},-\mathrm{OH}$, and $-\mathrm{NH}_{2}$ disrupt the avidity of this predicted $\mathrm{H}$-bonding interaction due to their $\pi$ donating properties through the aromatic ring.

In summary, a series of $\mathrm{N}, \mathrm{N}$-diphenylamine derivatives have been designed, synthesized, and evaluated systematically as EPAC2-specific inhibitors. Molecular docking was further used to provide insight into potential binding modes. Specifically, an allosteric site unique to EPAC2 was identified and the predicted ligand-protein interactions were used to rationalize the observed SAR data and should stimulate new strategies toward the inhibition of EPAC proteins. Compounds 12 (CTW0151), 15 (MAY0132), 27 (CTW0181), and 31 (CTW0167) displayed no substantial activity at the highly homologous
EPAC1 while exhibiting submicromolar potency at EPAC2. To the best of our knowledge, compounds 12, 15, 27, and 31 are comparable with the most potent of any synthesized EPAC2 selective inhibitors reported to date. These compounds, with decent physicochemical profiles (i.e., avg ligand efficiency $=$ 0.41. avg tPSA $=13$, avg $\mathrm{Mw}=268$, avg $\operatorname{Clog} \mathrm{P}=5.4$; see SI, Table S1), may be useful in facilitating our efforts in uncovering the isoform-specific roles of EPAC proteins. Further studies to improve the drug-likeness (e.g., lipophilicity and solubility) of these molecules while maintaining activity/selectivity are currently underway.

\section{ASSOCIATED CONTENT}

\section{S Supporting Information}

The Supporting Information is available free of charge on the ACS Publications website at DOI: 10.1021/acsmedchemlett.5b00477.

Detailed experimental procedures, spectroscopic data of all compounds, and methods of biological evaluation (PDF)

\section{AUTHOR INFORMATION}

\section{Corresponding Authors}

*(X.C.) Tel: (713) 500-7487. Fax: (409) 500-7465. E-mail: xiaodong.cheng@uth.tmc.edu.

*(J.Z.) Tel: (409) 772-9748. Fax: (409) 772-9648. E-mail: jizhou@utmb.edu.

\section{Author Contributions}

${ }^{\S}$ These authors contributed equally to this work and should be considered as co-first authors.

\section{Funding}

This work was supported by grants R01 GM066170, GM106218, and F31 DA038922-01A1 from the National Institutes of Health, a training fellowship from the Keck Center for Interdisciplinary Bioscience Training of the Gulf Coast Consortia (GCC) (NIGMS grant T32 GM089657), and the Center for Addiction Research (NIDA grant T32 DA07287).

\section{Notes}

The authors declare no competing financial interest.

\section{ACKNOWLEDGMENTS}

We would like to thank Drs. Lawrence C. Sowers and Jacob A. Theruvathu at the Department of Pharmacology and Dr. Tianzhi Wang at the NMR core facility of UTMB for the NMR spectroscopy assistance.

\section{ABBREVIATIONS}

EPAC, exchange protein directly activated by cAMP; cAMP, cyclic adenosine monophosphate; PKA, protein kinase A; HTS, high-throughput screening; SARs, structure-activity relationships; GEF, guanine nucleotide exchange factor; CNBD, cyclic nucleotide binding domain; 8-NBD-cAMP, 8-(2-[7-nitro-4benzofurazanyl $]$ aminoethylthio)adenosine- $3^{\prime}, 5^{\prime}$-cyclic monophosphate; $\mathrm{Pd}_{2}(\mathrm{dba})_{3}$, tris(dibenzylidene-acetone)dipalladium(0); $\mathrm{NaO}^{t} \mathrm{Bu}$, sodium tert-butoxide; ( \pm )-BINAP, 2,2' -bis(diphenylphosphino)-1,1'-binaphthyl

\section{REFERENCES}

(1) Berman, H. M.; Ten Eyck, L. F.; Goodsell, D. S.; Haste, N. M.; Kornev, A.; Taylor, S. S. The cAMP binding domain: an ancient signaling molecule. Proc. Natl. Acad. Sci. U. S. A. 2005, 102, 45-50. 
(2) de Rooij, J.; Zwartkruls, F. J. T.; Verheijen, M. H. G.; Cool, R. H.; Nijman, S. M. B.; Wittinghofer, A.; Bos, J. L. Epac is a Rap1 guaninenucleotide-exchange factor directly activated by cyclic AMP. Nature 1998, 396, 474-477.

(3) Kawasaki, H.; Springett, G. M.; Mochizuki, N.; Toki, S.; Nakaya, M.; Matsuda, M.; Housman, D. E.; Graybiel, A. M. A Family of cAMPBinding Proteins That Directly Activate Rap1. Science 1998, 282, 2275-2279.

(4) Cheng, X.; Ji, Z.; Tsalkova, T.; Mei, F. Epac and PKA: a tale of two intracellular cAMP receptors. Acta Biochim. Biophys. Sin. 2008, 40, $651-662$.

(5) Dekkers, B. G.; Racke, K.; Schmidt, M. Distinct PKA and Epac compartmentalization in airway function and plasticity. Pharmacol. Ther. 2013, 137, 248-265.

(6) Banerjee, U.; Cheng, X. Exchange proteins directly activated by cAMP encoded by the mammalian rapgef3 gene: structure, function, and therapeutics. Gene 2015, 570, 157-167.

(7) Chen, H.; Wild, C.; Zhou, X.; Ye, N.; Cheng, X.; Zhou, J. Recent advances in the discovery of small molecules targeting exchange proteins directly activated by cAMP (EPAC). J. Med. Chem. 2014, 57, 3651-65.

(8) Almahariq, M.; Chao, C.; Mei, F. C.; Hellmich, M. R.; Patrikeev, I.; Motamedi, M.; Cheng, X. Pharmacological inhibition and genetic knockdown of exchange protein directly activated by cAMP 1 reduce pancreatic cancer metastasis in vivo. Mol. Pharmacol. 2015, 87, 142-9. (9) Almahariq, M.; Tsalkova, T.; Mei, F. C.; Chen, H.; Zhou, J.; Sastry, S. K.; Schwede, F.; Cheng, X. A novel EPAC-specific inhibitor suppresses pancreatic cancer cell migration and invasion. Mol. Pharmacol. 2013, 83, 122-8.

(10) Gong, B.; Shelite, T.; Mei, F. C.; Ha, T.; Hu, Y.; Xu, G.; Chang, Q.; Wakamiya, M.; Ksiazek, T. G.; Boor, P. J.; Bouyer, D. H.; Popov, V. L.; Chen, J.; Walker, D. H.; Cheng, X. Exchange protein directly activated by cAMP plays a critical role in bacterial invasion during fatal rickettsioses. Proc. Natl. Acad. Sci. U. S. A. 2013, 110, 19615-20.

(11) Tao, X.; Mei, F.; Agrawal, A.; Peters, C. J.; Ksiazek, T. G.; Cheng, X.; Tseng, C. T. Blocking of exchange proteins directly activated by cAMP leads to reduced replication of Middle East respiratory syndrome coronavirus. J. Virol. 2014, 88, 3902-10.

(12) Almahariq, M.; Mei, F. C.; Cheng, X. Cyclic AMP sensor EPAC proteins and energy homeostasis. Trends Endocrinol. Metab. 2014, 25, $60-71$.

(13) Yan, J.; Mei, F. C.; Cheng, H.; Lao, D. H.; Hu, Y.; Wei, J.; Patrikeev, I.; Hao, D.; Stutz, S. J.; Dineley, K. T.; Motamedi, M.; Hommel, J. D.; Cunningham, K. A.; Chen, J.; Cheng, X. Enhanced leptin sensitivity, reduced adiposity, and improved glucose homeostasis in mice lacking exchange protein directly activated by cyclic AMP isoform 1. Mol. Cell. Biol. 2013, 33, 918-26.

(14) Ulucan, C.; Wnag, X.; Balijinnyam, E.; Bai, Y.; Okumura, S.; Sato, M.; Minamisawa, S.; Hirotani, S.; Ishikawa, Y. Developmental changes in gene expression of Epac and its upregulation in myocardial hypertrophy. Am. J. Physiol. Heart Cir. Physiol. 2007, 293, H1662H1672.

(15) Metrich, M.; Lucas, A.; Gastineau, M.; Samuel, J. L.; Heymes, C.; Morel, E.; Lezoualc'h, F. Epac mediates beta-adrenergic receptorinduced cardiomyocyte hypertrophy. Circ. Res. 2008, 102, 959-65.

(16) de Rooij, J.; Rehmann, H.; van Triest, M.; Cool, R. H.; Wittinghofer, A.; Bos, J. L. Mechanism of regulation of the Epac family of cAMP-dependent RapGEFs. J. Biol. Chem. 2000, 275, 20829-36.

(17) Zhu, Y.; Chen, H.; Boulton, S.; Mei, F.; Ye, N.; Melacini, G.; Zhou, J.; Cheng, X. Biochemical and Pharmacological Characterizations of ESI-09 Based EPAC Inhibitors: Defining the ESI-09 "Therapeutic Window". Sci. Rep. 2015, 5, 9344.

(18) Tsalkova, T.; Fang, M. C.; Cheng, X. A Fluorescence-Based High-Throughput Assay for the Discovery of Exchange Protein Directly Activated by Cyclic AMP (EPAC) Antagonists. PLoS One 2012, 7, e30441.

(19) Chen, H.; Tsalkova, T.; Mei, F. C.; Hu, Y.; Cheng, X.; Zhou, J. 5-Cyano-6-oxo-1,6-dihydro-pyrimidines as potent antagonists target- ing exchange proteins directly activated by cAMP. Bioorg. Med. Chem. Lett. 2012, 22, 4038-43.

(20) Ye, N.; Zhu, Y.; Chen, H.; Liu, Z.; Mei, F. C.; Wild, C.; Chen, H.; Cheng, X.; Zhou, J. Structure activity relationship of substituted 2(isoxazol-3-yl)-2-oxo- $\mathrm{N}^{\prime}$-phenyl-acetohydrazonoyl cyanide analogues: identification of potent exchange proteins directly activated by cAMP (EPAC) antagonists. J. Med. Chem. 2015, 58, 6033-6047.

(21) Tsalkova, T.; Mei, F. C.; Li, S.; Chepurny, O. G.; Leech, C. A.; Liu, T.; Holz, G. G.; Woods, V. L., Jr.; Cheng, X. Isoform-specific antagonists of exchange proteins directly activated by cAMP. Proc. Natl. Acad. Sci. U. S. A. 2012, 109, 18613-8.

(22) Chen, H.; Tsalkova, T.; Chepurny, O. G.; Mei, F. C.; Holz, G. G.; Cheng, X.; Zhou, J. Identification and characterization of small molecules as potent and specific EPAC2 antagonists. J. Med. Chem. 2013, 56, 952-62.

(23) Chen, H.; Ding, C.; Wild, C.; Liu, H.; Wang, T.; White, M. A.; Cheng, X.; Zhou, J. Efficient Synthesis of ESI-09, A Novel Non-cyclic Nucleotide EPAC Antagonist. Tetrahedron Lett. 2013, 54, 1546-1549.

(24) Rehmann, H.; Das, J.; Knipscheer, P.; Wittinghofer, A.; Bos, J. Structure of the cyclic-AMP-responsive exchange factor Epac2 in its auto-inhibited state. Nature 2006, 439, 625-8.

(25) Li, S.; Tsalkova, T.; White, M. A.; Mei, F. C.; Liu, T.; Wang, D.; Woods, V. L., Jr; Cheng, X. Mechanism of intra-cellular cAMP sensor Epac2 activation: cAMP-induced conformational changes identified by amide hydrogen/deuterium exchange mass spectrometry (DXMS). J. Biol. Chem. 2011, 286, 17889-17897.

(26) Selvaratnam, R.; VanSchouwen, B.; Fogolari, F.; Mazhab-Jafari, M. T.; Das, R.; Melacini, G. The projection analysis of NMR chemical shifts reveals extended EPAC autoinhibition determinants. Biophys. J. 2012, 102, 630-639.

(27) Selvaratnam, R.; Mazhab-Jafari, M. T.; Das, R.; Melacini, G. The auto-inhibitory role of the EPAC hinge helix as mapped by NMR. PLoS One 2012, 7, e48707.

(28) Akimoto, M.; Selvaratnam, R.; McNicholl, E. T.; Verma, G.; Taylor, S. S.; Melacini, G. Signaling through dynamic linkers as revealed by PKA. Proc. Natl. Acad. Sci. U. S. A. 2013, 110, 1423114236.

(29) Boulton, S.; Akimoto, M.; Selvaratnam, R.; Bashiri, A.; Melacini, G. A toll set to map allosteric networks through the NMR chemical shift covariance analysis. Sci. Rep. 2014, 4, 7306.

(30) Brock, M.; Fan, F.; Mei, F. C.; Li, S.; Gessner, C.; Woods, V. L., $\mathrm{Jr}$; Cheng, X. Conformational analysis of Epac activation using amide hydrogen/deuterium exchange mass spectrometry. J. Biol. Chem. 2007, 282, 32256-32263.

(31) Rehmann, H. Epac-Inhibitors: facts and artefacts. Sci. Rep. 2013, 3, 3032 .

(32) Brown, L. M.; Rogers, K. E.; Aroonsakool, N.; McCammon, J. A.; Insel, P. A. Allosteric inhibitors of Epac, computational modeling and experimental validation to identify allosteric sites and inhibitors. J. Biol. Chem. 2014, 289, 29148-29157.

(33) Courilleau, D.; Bisserier, M.; Jullian, J.; Lucas, A.; Bouyssou, P.; Fischmeister, R.; Blondeau, J.; Lezoualc'h, F. Identification of a tetrahydroquinoline analog as a pharmacological inhibitor of the cAMP-binding protein EPAC. J. Biol. Chem. 2012, 287, 44192-44202. 